\title{
Regulation of REG $\gamma$ cellular distribution and function by SUMO modification
}

Yan $\mathrm{Wu}^{1,{ }^{*}}$, Lu Wang ${ }^{1, *}$, Ping Zhou ${ }^{1}$, Guangqiang Wang ${ }^{1}$, Yu Zeng ${ }^{1}$, Ying Wang ${ }^{1}$, Jian Liu ${ }^{1,3}$, Bianhong Zhang ${ }^{1}$, Shuang Liu ${ }^{1}$, Honglin $\mathrm{Luo}^{2}$, Xiaotao $\mathrm{Li}^{1,3}$

${ }^{1}$ Institute of Biomedical Sciences, East China Normal University, 500 Dongchuan Road, Shanghai 200241, China; ${ }^{2}$ The James Hogg iCAPTURE Centre for Cardiovascular and Pulmonary Research, University of British Columbia-St Paul's Hospital, 1081 Burrard Street, Vancouver, Canada V6Z 1Y6; ${ }^{3}$ Department of Molecular and Cellular Biology, Baylor College of Medicine, One Baylor Plaza, Houston, TX 77030, USA

Discovery of emerging REG $\gamma$-regulated proteins has accentuated the REG $\gamma$-proteasome as an important pathway in multiple biological processes, including cell growth, cell cycle regulation, and apoptosis. However, little is known about the regulation of the REG $\gamma$-proteasome pathway. Here we demonstrate that REG $\gamma$ can be SUMOylated in vitro and in vivo by SUMO-1, SUMO-2, and SUMO-3. The SUMO-E3 protein inhibitor of activated STAT (PIAS)1 physically associates with REG $\gamma$ and promotes SUMOylation of REG $\gamma$. SUMOylation of REG $\gamma$ was found to occur at multiple sites, including K6, K14, and K12. Mutation analysis indicated that these SUMO sites simultaneously contributed to the SUMOylation status of REG $\gamma$ in cells. Posttranslational modification of REG $\gamma$ by SUMO conjugation was revealed to mediate cytosolic translocation of REG $\gamma$ and to cause increased stability of this proteasome activator. SUMOylation-deficient REG $\gamma$ displayed attenuated ability to degrade $p 21^{\text {Wat//ip1 }}$ due to reduced affinity of the REG $\gamma$ SUMOylation-defective mutant for $\mathbf{p 2 1}$. Taken together, we report a previously unrecognized mechanism regulating the activity of the proteasome activator REG $\gamma$. This regulatory mechanism may enable REG $\gamma$ to function as a more potent factor in protein degradation with a broader substrate spectrum.

Keywords: REG $\gamma$; SUMO; modification; PIAS1

Cell Research (2011) 21:807-816. doi:10.1038/cr.2011.57; published online 29 March 2011

\section{Introduction}

The proteasome activator REG $\gamma$ (also known as PA28 $\gamma$, PSME3, Ki antigen) has been shown to promote degradation of intact cellular proteins such as the nuclear receptor cofactor SRC-3 and cyclin-dependent kinase inhibitor p21 [1-3]. Aberrant expression of REG $\gamma$ is involved in cancer progression $[4,5]$. A recent comparative analysis of REG $\gamma$ expression in mouse and human tissues revealed unique patterns of REG $\gamma$ in specific cell types, suggesting additional undisclosed functions and biological importance of this molecule [6]. How REG $\gamma$ function

*These two authors contributed equally to this work. Correspondence: Xiaotao Li

Tel: +713-7983817; Fax: 713-7901275

E-mail: xiaotaol@bcm.edu

Received 4 August 2010; revised 2 November 2010; accepted 24 November 2010; published online 29 March 2011 is regulated within mammalian cells is unclear.

The Small Ubiquitin-related Modifier or SUMO proteins, including the ubiquitously expressed SUMO1, SUMO-2, and SUMO-3, are a family of ubiquitinlike polypeptides, which can be covalently attached to target proteins. Posttranslational SUMO modification of proteins results in modification of their functions, such as nuclear-cytosolic transportation, protein-protein interactions, protein stability, and modulation of cellular processes such as response to stress or cell cycle progression. Similar to the ubiquitination process, SUMOylation also requires activating (E1), conjugating (E2), and ligating (E3) enzymatic steps. In contrast to the ubiquitin pathway, SUMOylation process is mediated by a single E2 enzyme, UBC9, and a limited number of E3 enzymes [7]. Among the recognized SUMO ligases, proteins with Siz/PIAS RING-finger-like domains (SP-RING domains) are known as protein inhibitor of activated STAT (PIAS) proteins in vertebrates [8]. The five vertebrate PIAS 
proteins (PIAS1, PIAS3, PIASx $\alpha$, PIASx $\beta$, and PIASy) have been implicated in many processes, including gene expression, signal transduction, and genome maintenance [9]. Yet, their biological targets are not fully disclosed.

Here we report that the proteasome activator REG $\gamma$ can be SUMOylated posttranslationally by PIAS 1 . SUMO modification of REG $\gamma$ at specific sites results in cytoplasmic localization and increased stability of REG $\gamma$. SUMOylation-defective mutant REG $\gamma$ is attenuated for its function in degrading its target protein, $\mathrm{p} 21^{\text {Waf//Cip1 }}$. These results reveal a previously unknown mechanism for the regulation of REG $\gamma$ activity.

A

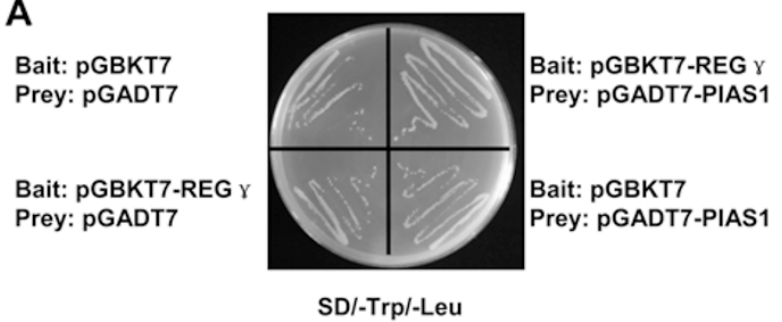

B

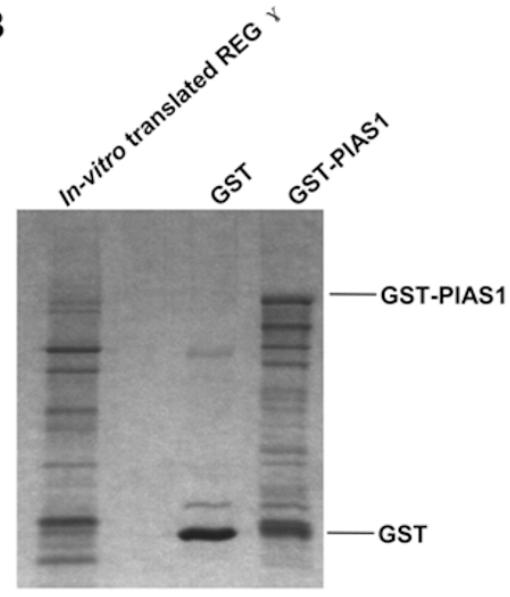

Commassie Stain

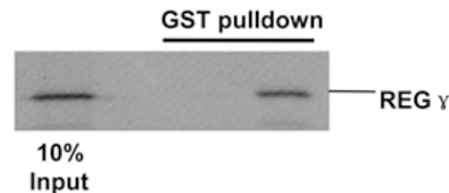

\section{Results}

Identification of PIAS1 as a REG $\gamma$ interacting protein

In an effort to search for REG $\gamma$ interacting proteins, we performed yeast two-hybrid analysis using REG $\gamma$ as the bait. One of the REG $\gamma$ interacting proteins is PIAS1. We verified the physical interaction between REG $\gamma$ and PIAS1 in yeast strain AH109 (Clontech) with a medium lacking Ade/His/Trp/Leu for quadruplicate selection of growth (Figure 1A). To further validate this interaction, we constructed GST-PIAS1 and purified GST and GSTPIAS1 proteins from bacteria (Figure 1B, upper panel).

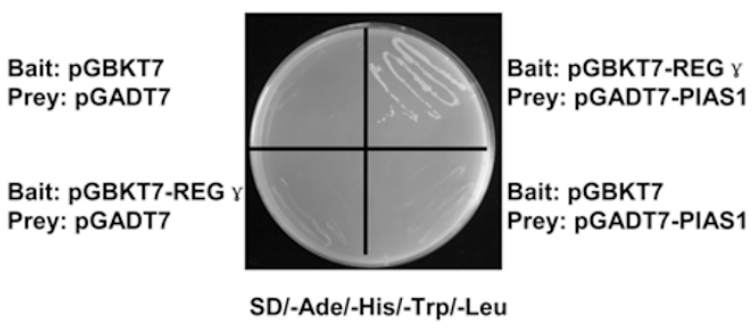

C

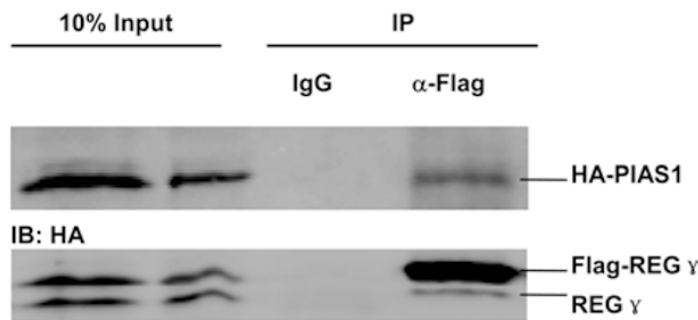

IB: REG Y

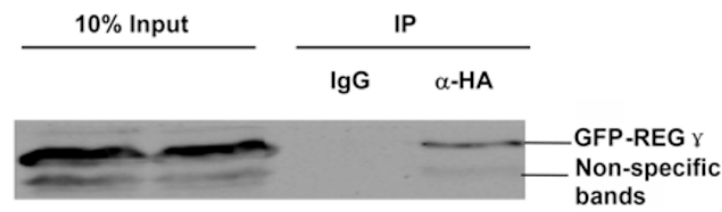

IB: REG Y

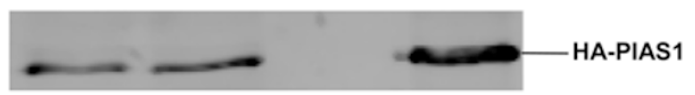

IB: HA

Figure 1 REG $\gamma$ interacts with PIAS1 in vitro and in vivo. (A) Interaction of REG $\gamma$ and PIAS1 in yeast. AH109 was transformed with the indicated bait and prey plasmids as described in Materials and Methods. The transformants were then streaked on SD/-Trp/-Leu (Left) to indicate proper expression of each construct and a replicate on SD/-Ade/-His/-Trp/-Leu (right) selective plate demonstrated the physical interaction between REG $\gamma$ and PIAS1. (B) REG $\gamma$ interacts with PIAS1 in vitro. GST-PIAS1 or GST only was incubated with in vitro-translated REG $\gamma$ as described in Materials and Methods. The relative amounts of GST and GST fusion proteins are shown in the Coomassie-stained gel (upper panel). Following 2-h incubation at $4{ }^{\circ} \mathrm{C}$, the complex was pulled down by GST beads, and REG $\gamma$ level was detected by autoradiograph (lower panel). (C) REG $\gamma$ interacts with PIAS1 in cells. 293T cells were co-transfected with HA-PIAS1 and Flag-REG $\gamma$ or GFP-REG $\gamma$ for $48 \mathrm{~h}$ as described in Materials and methods. Immunoprecipitation was performed with whole cell lysates using anti-flag antibody, anti-HA or lgG (control). The immunocomplexes were separated by SDS-PAGE and detected by an antibody against REG $\gamma$ or HA. 
GST pull-down experiments were carried out in the presence of in vitro translated REG $\gamma$. Compared to GST alone, GST-PIAS1 can pull down a remarkable amount of REG $\gamma$ (Figure 1B, lower panel). We then tested the intracellular capability of REG $\gamma$ to interact with PIAS1. By overexpressing both REG $\gamma$ and PIAS1 in 293T cells and immunoprecipitating REG $\gamma$, we detected overt coimmunoprecipitation of PIAS1 (Figure 1C, upper panel). We also performed reciprocal experiments by immunoprecipitating PIAS1 to detect co-immunoprecipitation of REG $\gamma$. Taken together, these experiments support that REG $\gamma$ directly interacts with PIAS1.

PIAS1 promotes SUMO-modification of REG $\gamma$ in cells

To test if REG $\gamma$ can be SUMOylated, we first per- formed in vitro SUMOylation study in the presence of SUMO E1 (AOS1/UBA2), SUMO E2 (UBC9), and ATP. Figure 2A demonstrates that SUMO-1, -2 , and -3 can be utilized in vitro to generate multiple forms of SUMOylated REG $\gamma$. To further verify REG $\gamma$ SUMOylation in vivo, we performed cell-based analysis to enrich SUMOREG $\gamma$ conjugates by purification of 6His-SUMO under denaturing conditions, followed by western blot with anti-REG $\gamma$. To enhance the assay sensitivity, we used GFP-REG $\gamma$ as the substrate, which does not form a homoheptamer. While GFP protein could not be SUMOylated (Supplementary information, Figure S1, lanes 3 and 4), GFP-REG $\gamma$ was heavily SUMOylated in the presence of wild type (wt) His-SUMO-2 and His-SUMO-3 (Figure 2B, lanes 2 and 4; Supplementary information, Figure

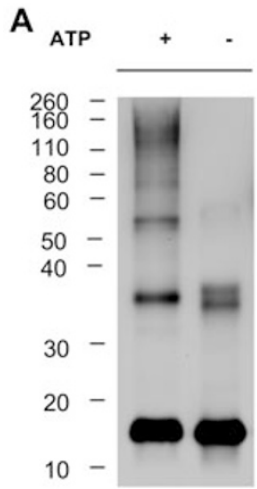

SUMO-1

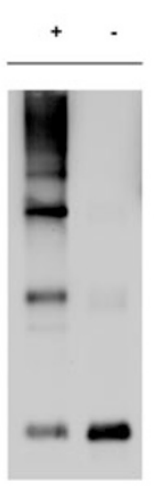

SUMO-2

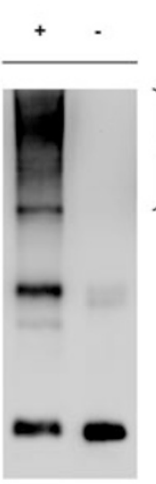

SUMO-3

B

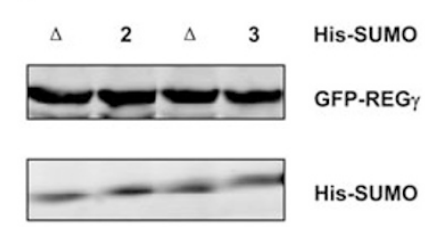

$5 \%$ input

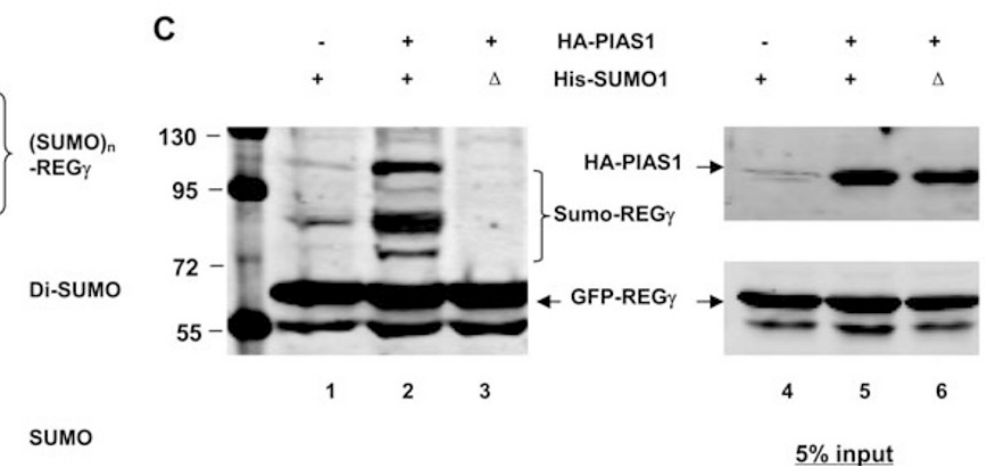

$5 \%$ input
.

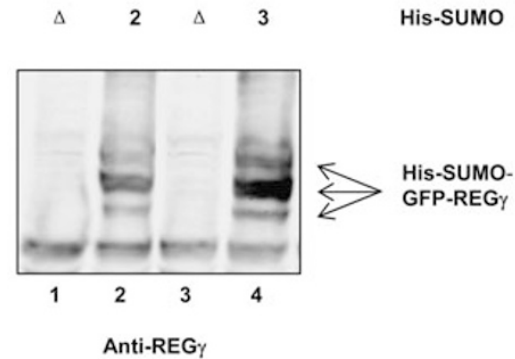

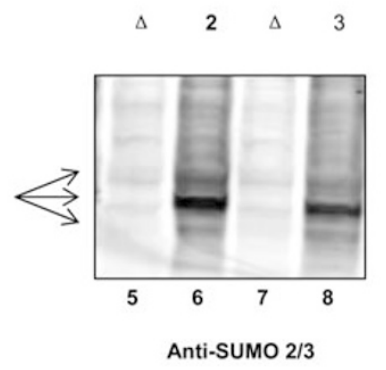

Figure $2 \mathrm{REG} \gamma$ is SUMOylated in vitro and in vivo. (A) SUMOylation of REG $\gamma$ in vitro. In vitro SUMOylation assay was carried out as described in Materials and methods in a reaction buffer with (+) or without (-) ATP. The reaction mixtures were analyzed by western blot with antibodies to SUMO-1 and SUMO-2/3, respectively. (B) SUMOylation of REG $\gamma$ in vivo. 293T cells were co-transfected with GFP-REG $\gamma$ or GFP, along with Ubc9, His-SUMO-2, or conjugation-deficient His-SUMO-2 ( $\triangle$ GG) mutant. SUMO substrates were purified using Ni-NTA-agarose beads and probed with REG $\gamma$ antibodies as described in Materials and methods. The left panel displays the input of GFP-REG $\gamma$, His-SUMO-2/3, and defective His-SUMO-2/3 ( $\Delta$ GG) as control. The middle panel shows the His-SUMO-GFP-REG $\gamma$ conjugates detected by western blot using anti-REG $\gamma$. The right panel shows His-SUMO-GFP-REG $\gamma$ conjugates (pointed with arrows) detected with anti-SUMO 2/3. No SUMO conjugation was observed in defective His-SUMO-2/3 ( $\triangle \mathrm{GG}$ ) transfected controls. (C) PIAS1 enhances SUMOylation of REG $\gamma$. 293T cells were transfected with GFP-REG $\gamma$, His-SUMO1, or His-SUMO-1 $(\Delta G G)$ in the presence or absence of HA-PIAS1. SUMOylated protein was enriched with Ni-NTA-agarose beads and probed with REG $\gamma$ antibody. The left panel displays marked increase of SUMOylated GFP-REG $\gamma$ in the presence of HA-PIAS1, but no SUMOylation in His-SUMO-1 ( $\triangle \mathrm{GG)}$ transfected control. The input expressions of HA-PIAS1 and GFP-REG $\gamma$ are shown in the right panel. 
S1, lane 1), but not when conjugation-defective HisSUMO-2/3 $(\Delta \mathrm{GG})$ were coexpressed (Figure $2 \mathrm{~B}$, lanes 1 and 3). To validate if the SUMO-GFP-REG $\gamma$ complexes detected in Figure 2B by western blot with a rabbit antiREG $\gamma$ were truly conjugates of SUMO proteins, the same membrane was probed with a mouse anti-SUMO $2 / 3$. Similar patterns of SUMO-GFP-REG $\gamma$ complexes were observed in Figure 2B, lanes 6 and 8, but not in the presence of conjugation-defective His-SUMO-2/3 (Figure 2B, lanes 5 and 7). We also observed that SUMO-1 can be conjugated to the REG $\gamma$ molecule in cells (Supplementary information, Figure S2).

PIAS1 has been shown to have SUMO-E3 activity [9]. Given the evidence for physical interaction between PIAS1 and REG $\gamma$, we investigated if PIAS1 can promote SUMO-modification of REG $\gamma$ in vivo. GFP-REG $\gamma$ was coexpressed with His-SUMO-1/His-SUMO-1 ( $\Delta \mathrm{GG})$, HA-PIAS1 or a control vector, followed by enrichment of His-SUMO-REG $\gamma$ conjugates with affinity chromatography. The amount of SUMOylated REG $\gamma$ in the presence of exogenous PIAS1 was more than three-fold higher (Figure 2C, lanes 2) than that of the control sample (Figure 2C, lanes 1). To confirm that the slow migrating bands are SUMO-conjugated GFP-REG $\gamma$, the inactive His-SUMO-1 $(\Delta \mathrm{GG})$ was used as specificity control to show that no SUMOylation of REG $\gamma$ occurred under such condition (Figure 2C, lane 3). We further tested other PIAS family members to determine the specificity of PIAS1 as an E3 ligase for REG $\gamma$. Cells co-expressing PIAS1, PIAS3, PIASx $\alpha$, PIASx $\beta$ or PIASy were examined for SUMO conjugation of REG $\gamma$. Compared with control, only PIAS1 can promote robust SUMOylation of REG $\gamma$ despite that PIASx $\beta$ also had a minor effect on REG $\gamma$ modification (Supplementary information, Figure S3, lanes 2 and 4). These results indicate that PIAS1 functions as a specific SUMO-E3 for REG $\gamma$ modification in vivo.

SUMO modification results in cytoplasmic translocation of $R E G \gamma$

Protein SUMOylation has been known to regulate subcellular localization $[9,10]$. We explored the possibility that SUMOylated REG $\gamma$ may also have altered subcellular localization patterns. We first overexpressed exogenous REG $\gamma$ in wt MEF cells or in MEFs defective for SENP-1, which catalyzes deconjugation of SUMOcontaining species. While the wt MEFs transfected with REG $\gamma$ had $\sim 2 \%$ of cells with cytoplasmic localization (Figure 3A, upper panel), REG $\gamma$-transfected SENP-1 ${ }^{-/}$ MEFs showed $\sim 24 \%$ of cells with cytoplasmic staining of REG $\gamma$ by immunofluorescent image analysis (Figure $3 \mathrm{~A}$, lower panel). We further investigated the distribution of endogenous REG $\gamma$ in wt and SENP- $1^{-/-}$MEFs by immunostaining. Similar to exogenously expressed REG $\gamma$, endogenous REG $\gamma$ also showed cytoplasmic expression in a significantly higher percentage of SENP- $1^{-/}$MEF cells (Figure 3B, lower panel) than in wt MEFs, which had less than $2 \%$ of cells with cytoplasmic REG $\gamma$ staining (Figure 3B, upper panel). To further validate that cellular redistribution of REG $\gamma$ is regulated by SUMO-modification, we generated $\mathrm{K}$ to $\mathrm{R}$ mutations in six predicted SUMO sites in REG $\gamma$ (construct named REG $\gamma-6 \mathrm{KR}$ based on the information in Supplementary information, Table S1). GFP-REG $\gamma$ and GFP-REG $\gamma$-6KR were transfected into SENP- $1^{--}$MEF cells to investigate the cytoplasmic expression profiles. In line with our prediction, wt GFP-REG $\gamma$ displayed cytoplasmic staining in $~ 30 \%$ of transfected cells (Figure 3C). In contrast, less than $5 \%$ of cells revealed cytoplasmic distribution in cells transfected with SUMOylation-deficient GFP-REG $\gamma$ 6KR (Figure 3C). Furthermore, we fused SUMO to the C-terminus of REG $\gamma$. Compared with intact REG $\gamma$, both REG $\gamma$-SUMO- 1 and REG $\gamma$-SUMO-2 fusion proteins exhibited significant nuclear export (Supplementary information, Figure S4A and S4B). Our data demonstrate that SUMOylation of REG $\gamma$ clearly regulates cellular distribution of this proteasome activator.

\section{SUMOylation of REG $\gamma$ occurs on multiple sites}

Since the covalent SUMO modification of target proteins occurs via lysine residues $[11,12]$, we searched potential SUMOylation sites in REG $\gamma$ sequence with bioinformatic analysis. Our analysis with SUMOplot indicated six candidate SUMO modification sites bearing the highest scores (Supplementary information, Table S1), while additional sites with high score were found using program SUMOsp2.0 (Supplementary information, Table S2). All these high-score sites are evolutionarily conserved among vertebrates and some are conserved in worm and insect (Supplementary information, Figure S5). To estimate the contribution of each lysine in SUMO modification, we generated the individual mutation of each lysine $(\mathrm{K})$ to arginine $(\mathrm{R})$ and generated constructs containing simultaneous mutation of all $\mathrm{K}$ to $\mathrm{R}$ at positions $123,6,14,243,46$, and 195 (named 6KR, note that the individual K-R mutation at position 6 is K6R). While most REG $\gamma$ constructs with individual $\mathrm{K}$ mutation did not reveal significantly quantifiable difference in SUMO conjugation patterns compared with wt by western analysis, double or triple mutations mostly revealed obvious SUMOylation deficiency (Figure 4A and Supplementary information, Figure S6). Simultaneous mutation of all six lysines in REG $\gamma$ significantly attenuated SUMO modification of REG $\gamma$ in vivo, yet SUMOylation of REG $\gamma$ was 
A
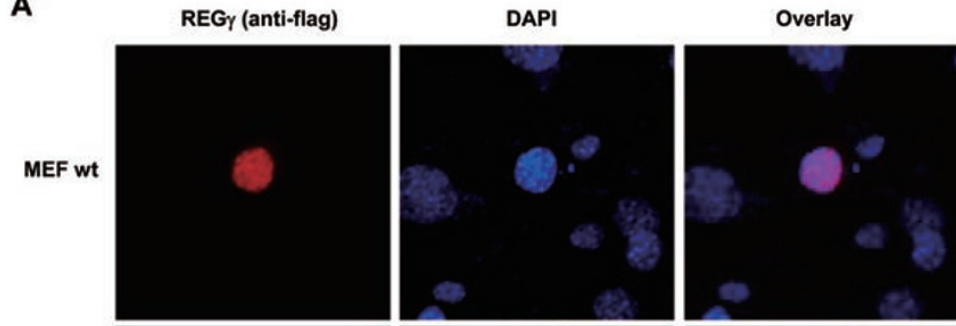

$2 \%, n=419$
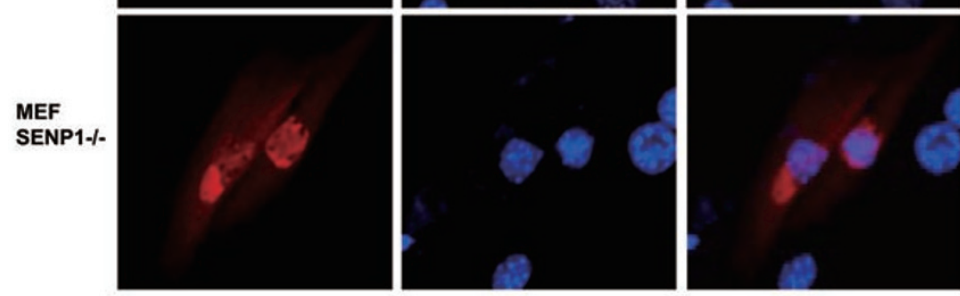

$24 \%, n=439$
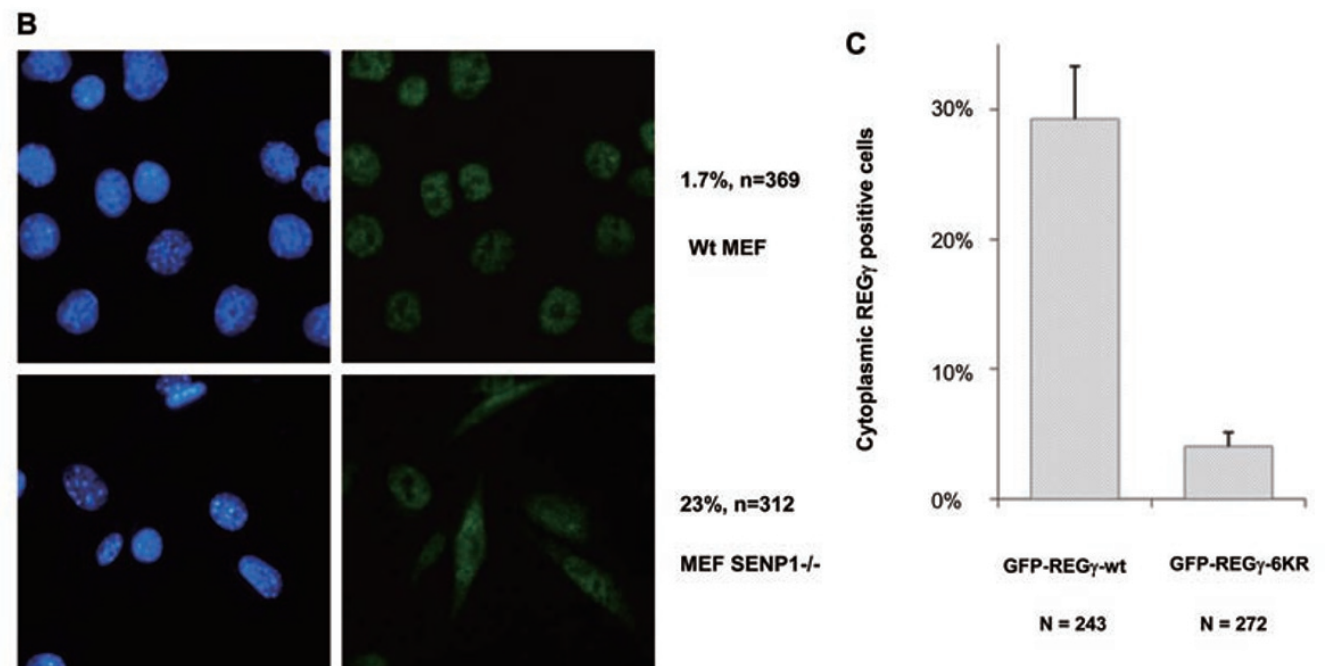

Figure 3 SUMO modification regulates cytosolic translocation of REG $\gamma$. (A) Cytosolic translocation of exogenous REG $\gamma$ in SUMOylation-active cells. Wild-type and SENP-1 ${ }^{-1-}$ MEF cells were transiently transfected with pcDNA5/FRT/TO Flag-REG $\gamma(1$ $\mu \mathrm{g})$. Twenty-four hours after transfection, immunostaining was performed with anti-DDK antibody (Flag) as described in Materials and methods to examine the expression and distribution of REG $\gamma$ (red). DAPI staining indicates the location of nuclei. The percentage of transfected cells with cytoplasmic expression of REG $\gamma$ is shown on the right. (B) SUMOylation enhances cytoplasmic translocation of endogenous REG $\gamma$. Wild-type and SENP-1 $1^{-1-}$ MEF cells were fixed and immunostaining was performed using anti-REG $\gamma$ as in (A) to visualize cellular localization of endogenous REG $\gamma$. The percentage of REG $\gamma$-positive cells with cytoplasmic translocation is shown on the right. (C) SUMOylation-deficient REG $\gamma$ has diminished cytoplasmic localization. SENP-1 ${ }^{-/-}$MEF cells were transfected with $1 \mu \mathrm{g}$ of GFP-REG $\gamma$ or GFP-REG $\gamma-6 \mathrm{KR}$ (mutation in all six predicated SUMO sites). The cytoplasmic vs nuclear fluorescent patterns in transfected cells expressing GFP fusion proteins were scored for statistical analysis. Data are shown as mean \pm SD of three independent experiments $(P<0.01)$.

not completely eradicated (Figure 4A, lanes 1 and 2), indicating additional sites being SUMOylated. These results suggest that REG $\gamma$ SUMOylation occurs in multiple sites.

To elucidate which of these prospective SUMO sites are important for SUMO modification of REG $\gamma$, we used a more quantifiable approach based on the fact that SUMOylated REG $\gamma$ can be exported to the cytoplasm. Transient transfection of each REG $\gamma$ construct with a single lysine mutation was scored for the percentage of cells with cytoplasmic localization of REG $\gamma$ in transfected SENP- $1^{-1-}$ MEF cells. Averaged results from three independent experiments suggested that compared to wt, REG $\gamma$ derivatives with K6R, K14R, and K12R mutations had significantly reduced capacity in cytoplasmic localization (Figure 4B), suggesting that each of these sites can be SUMOylated. To further examine the contribution of K12, K110, and K243 in REG $\gamma$ SUMOylation, we generated combination of triple mutations. Triple muta- 

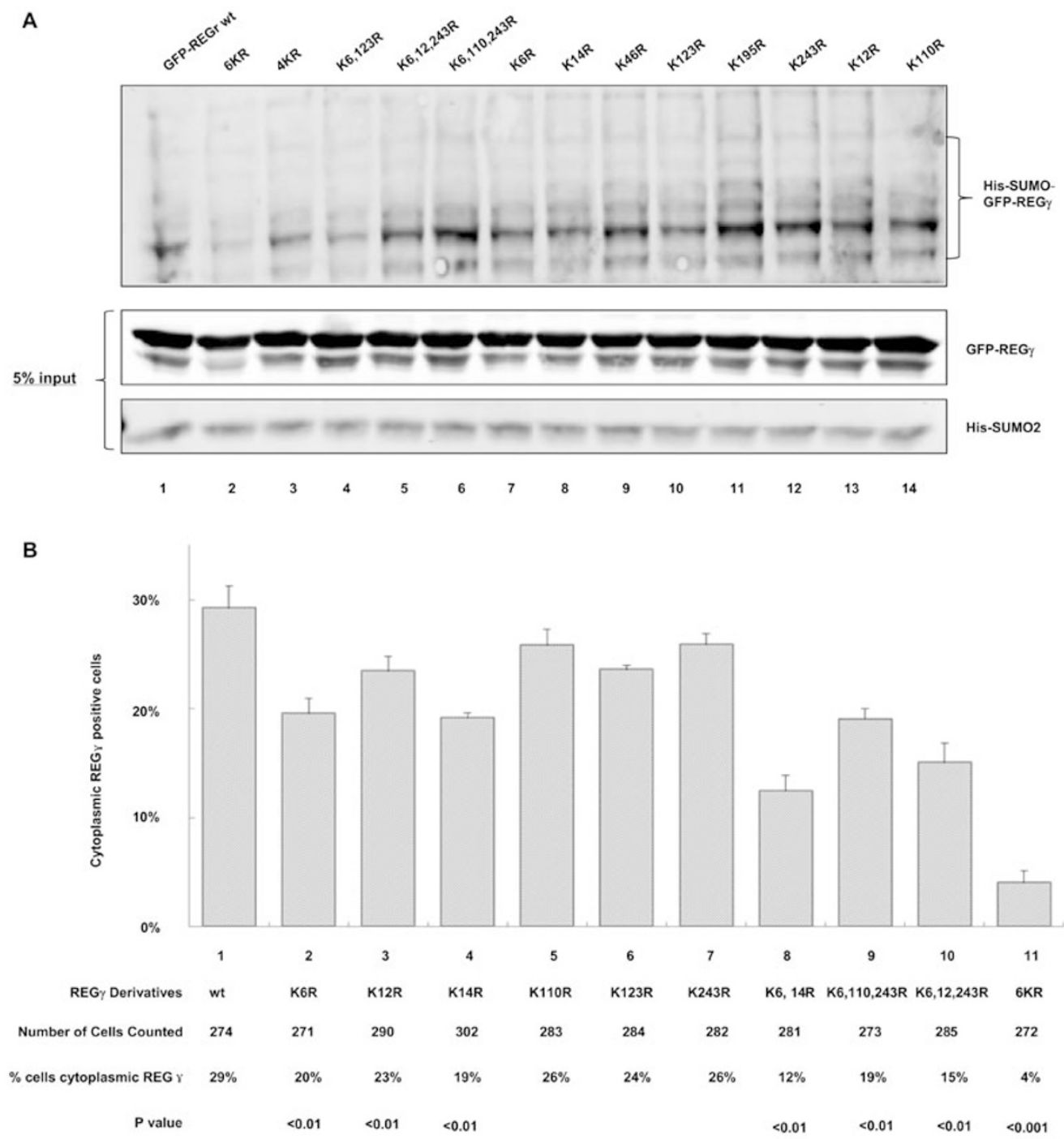

Figure 4 SUMOylation of REG $\gamma$ occurs on multiple sites. (A) REG $\gamma$ SUMOylation occurs on multiple sites. GFP-REG $\gamma$, GFPREG $\gamma-6 K R$, or GFP-REG $\gamma$ derivatives containing one or combinational mutations were co-expressed with His-SUMO-2 in 293T cells. SUMO conjugates were purified and examined as described in Materials and methods. GFP-REG $\gamma$-6KR (lane 2) had markedly reduced SUMO conjugates than GFP-REG $\gamma$ (lane 1). The 4KR refers to combinational mutations in K6, K12, $\mathrm{K} 110$, and K243. The input levels of His-SUMO-2 and GFP-REG $\gamma$ are shown in the lower panels. (B) Effect of SUMOylation site mutation(s) in cytosolic translocation of REG $\gamma$. Flag-REG $\gamma$ and Flag-REG $\gamma$ with single or combinatorial $\mathrm{K}$ mutation at putative SUMO site(s) were transfected into SENP-1 $1^{-1-}$ MEF cells. Twenty-four hours after transfection, cytoplasmic localization of transfected REG $\gamma$ derivatives was examined by immunostaining with anti-DDK (Flag) antibody and scored for statistical analysis. Data are shown as mean \pm SD of three independent experiments along with the number of cells counted and percentage of positive cells in the cytoplasm, as well as $P$ values.

tions in K6, K110, and K243R revealed no significant difference with that of K6R mutation alone, suggesting a less important role for K110 and K243 in SUMO modification. Compared with the K6 mutation, further reduction of cytoplasmic localization associated with REG $\gamma$ triple mutations at K6, K12, and K243R reflected a combinatorial contribution for K6 and K12 to SUMO modification of REG $\gamma$. Combined mutation of $\mathrm{K} 6$ and
$\mathrm{K} 14$ resulted in an even lower percentage in nuclear export than the triple mutation at K6, K12, and K243R. Consistent with SUMO-conjugation analysis, REG $\gamma$ with multiple mutations (REG $\gamma$-6KR) expressed in SENP-1 $1^{-1}$ MEF cells displayed a dramatic reduction in cytoplasmic localization (Figure 4A, lane 2 and Figure 4B, lane 11), supporting that SUMOylation of REG $\gamma$ occurs at multiple sites. 
SUMOylation enhances the stability of REG $\gamma$ protein in cells

A known consequence of SUMO modification is alteration of the stability of target proteins [9]. To test the effect of SUMO modification on REG $\gamma$ stability, we examined the decay rate of endogenous REG $\gamma$ in wt, and SENP-1 and SENP-2 knockout MEFs. Following treatment with cycloheximide (CHX), an inhibitor of protein biosynthesis in eukaryotic organisms, for 12 and $18 \mathrm{~h}$, the protein level of REG $\gamma$ was evaluated by western blot (Figure 5A). Results from three independent experiments were quantitated and averaged results were plotted in Figure 5B. Increased stability of REG $\gamma$ in SENP- $1^{-/}$MEFs was also observed with longer period of treatment by CHX (Supplementary information, Figure S7). Our results indicate that posttranslational modification of REG $\gamma$ by SUMOylation enhances the half-life of this proteasome activator in cells.

\section{SUMOylation-defective mutant of REG $\gamma$ has attenuated capacity to degrade $p 21$}

p21 has been reported as a direct substrate of REG $\gamma$ proteasome $[1,2]$. To understand the biological consequence of REG $\gamma$ SUMOylation, we analyzed the ability of SUMOylation-deficient REG $\gamma$-6KR to promote the degradation of $\mathrm{p} 21$. When wt REG $\gamma, \mathrm{REG} \gamma-6 \mathrm{KR}$, or an empty vector was co-expressed with $\mathrm{p} 21$ for $24 \mathrm{~h}$ to examine the activity of REG $\gamma$ in $\mathrm{p} 21$ degradation, we found a significant reduction in the capacity of REG $\gamma$ $6 \mathrm{KR}$ to degrade $\mathrm{p} 21$ (Figure 6A). To further validate the effect of REG $\gamma-6 \mathrm{KR}$ on $\mathrm{p} 21$ stability, we performed similar transient transfection experiments and treated cells with CHX for different periods of time to prevent de novo synthesis of proteins. We found faster decay of p21 in cells co-expressing wt REG $\gamma$ and much slower degradation of p21 in cells transfected with REG $\gamma$ $6 \mathrm{KR}$, suggesting an effect of REG $\gamma-6 \mathrm{KR}$ on $\mathrm{p} 21$ protein stability (Figure 6B). Consistent with this, we found that hyper-SUMOylated REG $\gamma$ in SENP-1 $1^{-/}$cells can promote endogenous p21 turnover. Despite the overall p21 levels in SENP- $1^{-/}$cells being higher, likely due to SUMOylation-mediated regulation of proteins like p53, we observed a faster decay rate of $\mathrm{p} 21$ in SENP-1 ${ }^{-/-}$cells (Supplementary information, Figure S8). Finally, we examined the potential mechanism involved in SUMOylation-mediated regulation of REG $\gamma$ activity. To test the impact of REG $\gamma$ SUMOylation on substrate binding, we expressed Flag-tagged REG $\gamma$ or SUMOylation-deficient REG $\gamma-6$ KR with p21 in 293 T cells. Following immunoprecipitation and western blotting analysis, we observed significantly reduced binding of SUMOylation-deficient REG $\gamma-6 \mathrm{KR}$ to $\mathrm{p} 21$. This may reflect the reduced ability

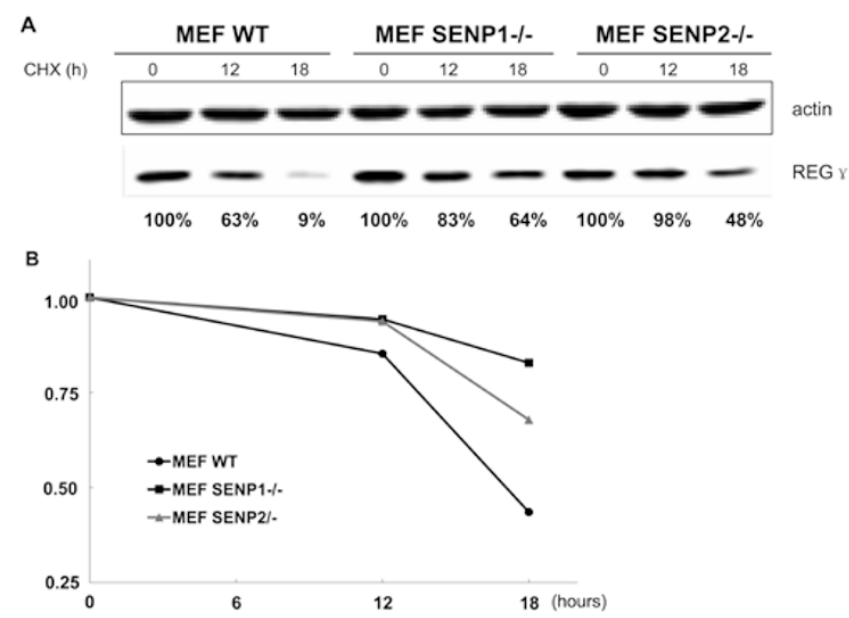

Figure 5 SUMOylation augments REG $\gamma$ stability. (A) REG $\gamma$ stability is increased in SENP $-1^{-1-}$ and SENP $-2^{-1-}$ MEFs. WT, SENP $-1^{-1-}$ and SENP-2 $2^{-1-}$ MEF cells were treated with cycloheximide $(100 \mu \mathrm{g} / \mathrm{ml})$ for the indicated time. An equal amount of total protein was loaded for western blotting analysis. (B) Quantitative analysis of REG $\gamma$ stability in SENP null MEFs. Experiments were performed as described in (A). Relative REG $\gamma$ level was plotted against the time course following cycloheximide treatment. Data are shown as mean of three independent experiments (standard deviation of the last time point is shown).

of REG $\gamma-6 \mathrm{KR}$ in recruiting $\mathrm{p} 21$ to the REG $\gamma$-proteasome for subsequent degradation.

\section{Discussion}

Along with the discovery of the first mammalian target of REG $\gamma$-proteasome [3], identification of increasing numbers of cellular proteins proteolytically regulated by REG $\gamma[1,2,13]$ has established the newly recognized alternative proteasome pathway. Yet, the regulatory input that may alter the biological function of REG $\gamma$ was not previously described. Here we show that SUMOylation of REG $\gamma$ can occur in vitro and in vivo. This posttranslational modification can be enhanced in the presence of PIAS1. SUMO modification of REG $\gamma$ mainly occurs at three lysine residues, which results in cytoplasmic distribution and stabilization of REG $\gamma$. We also demonstrated that the SUMOylation-defective mutant of REG $\gamma$ had attenuated capacity to degrade its target protein, p21.

To our knowledge, this study is the first report describing the molecular details of REG $\gamma$ SUMOylation, which can be facilitated by PIAS1. Our discovery that REG $\gamma$ physically associates with PIAS1 is consistent with a previously unpublished result mentioned in a review article [14]. Despite the SUMOylation of proteins being 
A

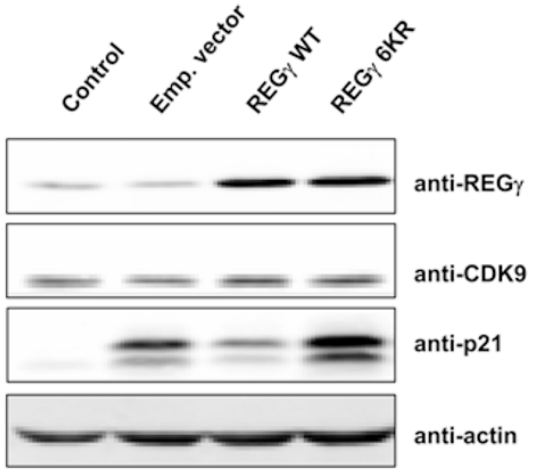

B $\begin{array}{lllllllll}\mathrm{CHX}(\mathrm{h}) & 0 & 1 & 2 & 3 & 0 & 1 & 2 & 3\end{array}$

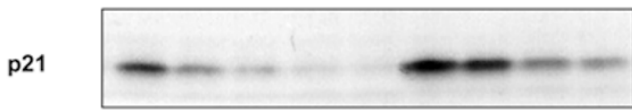

REG $\gamma$

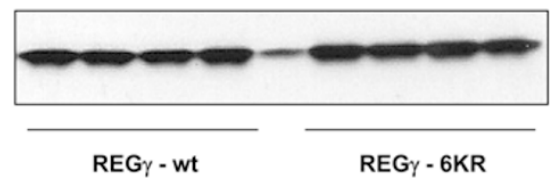

C

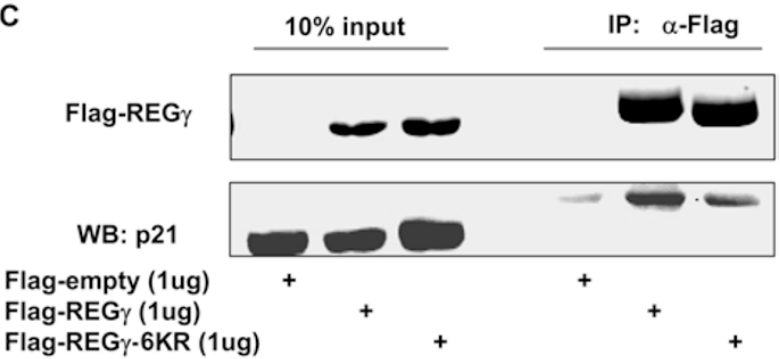

Figure 6 SUMOylation-deficient REG $\gamma$ has reduced activity in p21 degradation. (A) REG $\gamma-6 K R$ influences expression of p21. $\mathrm{H} 1299$ cells were transfected with REG $\gamma, \mathrm{REG} \gamma-6 \mathrm{KR}$, an empty vector, or a mock control along with p21 for $24 \mathrm{~h}$. Expression of the protein of interest was examined by western blotting with indicated antibodies. CDK9 serves as a control for specificity. (B) SUMOylation-defective REG $\gamma$ has attenuated proteolytic activity. Experiments were performed as in (A) and cells were treated with cycloheximide for the indicated time. The expression of p21 and dynamic decay rate were determined by western blot analysis. The expression of REG $\gamma$ and REG $\gamma-6 K R$ was equivalent in the two groups of samples examined. (C) SUMOylation-deficient $R E G \gamma-6 K R$ has attenuated affinity for $p 21$. In vivo interaction between p21 and wt or SUMOylation-defective REG $\gamma$ was examined by co-expressing these constructs and by subsequent immunoprecipitation analysis using anti-Flag antibody. Expression of a Flag-empty vector was used as negative control. To prevent degradation of the substrate p21, cells were treated with MG132 for $4 \mathrm{~h}$ before collection.

known to occur in the presence of SUMO-E1, E2, and SUMO in the absence of a SUMO-E3 [15], recent stud- ies have highlighted the importance of SUMO-E3, such as PIAS1, in numerous cases of SUMO modification of biologically important substrate proteins [16-18]. In our studies, we found that SUMO-1, -2 , and -3 can all be conjugated to REG $\gamma$ both in vitro and in vivo, suggesting that PIAS1-dependent and -independent processes are involved in REG $\gamma$ SUMOylation. Consistent with this notion, we observed that equivalent REG $\gamma$ SUMOylation occurred in both SENP-1 and SENP-2 knockout MEFs.

Although SUMO modification in some proteins occurs at a single site, SUMO conjugation via multiple sites was known to happen $[19,20]$. Our mutational analysis indicates that at least three lysines within REG $\gamma$ function as SUMO-acceptor residues. Notably, three of these residues, $\mathrm{K} 6, \mathrm{~K} 12$, and $\mathrm{K} 14$, are located within the $\mathrm{N}$-terminal region, which partially contributes to distinct functions between REG $\gamma$ and $\mathrm{REG} \alpha / \beta$ [4]. It is possible that SUMO modification in these three amino-acid residues is specific only to REG $\gamma$, but not to REG $\alpha / \beta$.

SUMO modification has been well known for regulation of cellular distribution of conjugated proteins. SUMO modification has been mostly described for its impact on nuclear import of target proteins. Nevertheless, SUMOylation also regulates nuclear export of modified proteins [21]. In our study, we found marked increase of cytoplasmic localization of SUMOylated REG $\gamma$, which normally stays in the nucleus. Therefore, SUMO modification enables REG $\gamma$ to reach substrates in the cytoplasm under certain conditions and increases the spectrum of REG $\gamma$-mediated protein degradation. In fact, we have analyzed the role of cytoplasmic REG $\gamma$ in coxsackieviral infection since coxsackievirus replication takes place exclusively in the cytoplasm. Relevant work is being published elsewhere.

Furthermore, we discovered that REG $\gamma$ SUMOylation enhanced its stability and that SUMOylation-deficient REG $\gamma$ had attenuated function in p21 degradation. Increased stability of endogenous REG $\gamma$ in SUMOylation super-active SENP-1 $1^{-/}$and SENP- $2^{-/}$cells is likely due to reduction of ubiquitination at the SUMO conjugated sites. Consistent with this prediction, we found that SUMOylation-deficient REG $\gamma$ is also more stable, probably due to ubiquitination deficiency as well (data not shown). Although increased stability of REG $\gamma$ may contribute to the rapid turnover of $\mathrm{p} 21$, enhanced REG $\gamma$ activity may play a more important role. The biological significance of REG $\gamma$ SUMOylation is highlighted by the observation that SUMOylation-defective REG $\gamma$ mutant shows significantly reduced ability to degrade $\mathrm{p} 21$. The observation of weakened physical interaction between the SUMOylation-defective REG $\gamma$ mutant and p21 further validated our hypothesis that perhaps posttranslational modifica- 
tion of REG $\gamma$ is required for this proteasome activator to fully interact with its substrate proteins or a specific target. Further experiments are required to determine whether other types of posttranslational modifications occur in $\mathrm{K} 6, \mathrm{~K} 14$, and $\mathrm{K} 12$, which may also contribute to the regulation of REG $\gamma$ function.

\section{Materials and Methods}

\section{Cell lines and cell culture}

HEK293T and H1299 cells were purchased from ATCC and maintained at Cell Culture Core in our Department. The SENP$1^{-/}$, SENP-2 ${ }^{-/-}$MEFs [22], SW1116 NS, and SW1116 SiSENP1 cells were kindly provided by Dr Jingke Chen from Shanghai Jiao Tong University School of Medicine. All cells were cultured under standard conditions described by the ATCC.

\section{Plasmids and reagents}

The cDNAs for SUMO-1, SUMO-2, and SUMO-3 were subcloned into pCNDA3.0 with N-terminally translational fusion of His-tags. SUMO- $1 \Delta \mathrm{GG}$ (deletion of C-terminal diglycine motif), SUMO2 $\Delta \mathrm{GG}$, and SUMO $3 \Delta \mathrm{GG}$ were generated by PCR mutagenesis. REG $\gamma$ and Flag-REG $\gamma$ from pCMV-Tag2B were subcloned into $\mathrm{pCNDA5/FRT/TO.} \mathrm{GFP-REG} \gamma$ was kindly provided by Domenico Delia at the Fondazione IRCCS Istituto Nazionale Tumori, Milano, Italy. Point mutants of REG $\gamma$ were created by PCR-based site-directed mutagenesis (Promega). Constructs expressing REG $\gamma$ SUMO-1 and REG $\gamma$-SUMO-2 fusion proteins were generated by two-step PCR strategy. GST-PIAS1 was generated by cloning a PCR product amplified from pXF3H-HA-PIAS1 [23] into pGEX4T-1.

Anti-HA and anti-GFP were purchased from Santa Cruz (sc7392 and sc-9996, respectively). Anti- $\beta$-actin was acquired from Sigma (A5441) and anti-DDK (also known as anti-Flag) from Origene (TA5011). REG $\gamma$ antibody was purchased from Invitrogen (38-3800) and monoclonal antibody against $\mathrm{p} 21$ was from BD Pharmingen (556431). Anti-Flag M2 Affinity Gel and Anti-HA Affinity Gel were from Sigma (F2426 and E6779, respectively). CHX was prepared as a $100-\mathrm{mg} / \mathrm{ml}$ stock solution dissolved in DMSO.

\section{Yeast two-hybrid analysis}

The full-length human REG $\gamma$ cDNA fragment was inserted in frame into the Gal4 DNA-binding domain (DBD) vector pGBKT7 and a human liver cDNA library (CLONTECH Laboratories, Inc.) was fused with the transcriptional activation domain of GAL4. We performed a medium-stringency scale procedure to screen REG $\gamma$ against human liver cDNA following the instructions of the manufacturer and characterized positive clones by sequence analysis.

\section{GST pull-down assays}

GST or GST-PIAS1 was purified as described [3] and preincubated with glutathione-sepharose beads. Then ${ }^{35} \mathrm{~S}$-Metlabeled, in vitro-translated REG $\gamma$ protein produced with a TNT kit (Promega) was incubated with the beads-bound GST derivatives at $4{ }^{\circ} \mathrm{C}$ for $2 \mathrm{~h}$. After extensive washing of the protein-bound beads with a buffer containing $20 \mathrm{mM}$ Tris-HCl, $\mathrm{pH} 7.4,200 \mathrm{mM}$ $\mathrm{NaCl}$, and $0.5 \%$ Triton X-100, PIAS1-bound REG $\gamma$ was separated by SDS-PAGE and visualized by autoradiography.

\section{Immunoprecipitation}

To test in vivo interactions between REG $\gamma$ and PIAS1, 293T cells were transfected with Flag-REG $\gamma(2 \mu \mathrm{g})$ and HA-PIAS1 (4 $\mu \mathrm{g})$ or HA-PIAS1 $(2 \mu \mathrm{g})$ along with GFP-REG $\gamma(4 \mu \mathrm{g})$ in $6-\mathrm{cm}$ dishes. Forty-eight hours after transfection, cells were harvested in ice-cold PBS and dispersed in lysis buffer (150 mM NaCl, $25 \mathrm{mM}$ Tris, $\mathrm{pH} 6.8,1 \% \mathrm{NP} 40)$. The lysates were centrifuged at $15000 \times \mathrm{g}$ for $20 \mathrm{~min}$ at $4{ }^{\circ} \mathrm{C}$. The cleared supernatant was mixed with $5 \mu 1$ of Anti-Flag M2 Affinity Gel or Anti-HA Affinity Gel and rotated for $4 \mathrm{~h}$ at $4{ }^{\circ} \mathrm{C}$. The immunoprecipitates were washed three times with NTN buffer (25 mM Tris, pH 6.8, $150 \mathrm{mM} \mathrm{NaCl}, 0.1 \%$ NP40). The washed beads were boiled with SDS sample buffer and subjected to SDS-PAGE analysis.

\section{Immunofluorescence}

Wild-type and SENP- $1^{-1-}$ MEFs were transfected with REG $\gamma$ derivative constructs, fixed, permeabilized, and immunoblotted as described previously [3]. Alexa Fluor 568 goat anti-mouse and Alexa Fluor 488 goat anti-rabbit secondary antibodies (Invitrogen) were used in epifluorescence microscopy. All image files were digitally processed for presentation with Image-Pro Plus and Adobe Photoshop.

\section{In vitro SUMOylation analysis}

The in vitro SUMOylation assay was carried out with a kit from Biomol (UW8955) using purified recombinant REG $\gamma$ (Biomol, PW9875) as a substrate. Briefly, purified REG $\gamma$ protein was incubated in a SUMOylation buffer with SUMOylation E1 (Aos1/ Uba2), E2 (Ubc9), and SUMO-1, -2 , or -3 in the presence or absence of $\mathrm{Mg}^{2+}$-ATP at $30{ }^{\circ} \mathrm{C}$ for $1 \mathrm{~h}$. The reaction products were separated by SDS-PAGE and analyzed by western blot using antiSUMO antibodies.

\section{In vivo SUMOylation assay}

To generate and purify His-tagged SUMO conjugates in vivo, $293 \mathrm{~T}$ cells were transfected with plasmids encoding GFP-REG $\gamma$, His-SUMO, or in combination with HA-PIAS1. Cells were harvested $36 \mathrm{~h}$ later and $5 \%$ of the cells were dispersed in lysis buffer $(150 \mathrm{mM} \mathrm{NaCl}, 25 \mathrm{mM}$ Tris-HCl, $\mathrm{pH}$ 6.8, $25 \mathrm{mM}$ Tris- $\mathrm{HCl}$, $\mathrm{pH} 8.0,1 \% \mathrm{NP} 40)$ as input. The rest was lysed in buffer A (6 M guanidinium- $\mathrm{HCl}, 0.1 \mathrm{M} \mathrm{Na} \mathrm{HPO}_{4} / \mathrm{NaH}_{2} \mathrm{PO}_{4}, \mathrm{pH} 8.0,0.01 \mathrm{M}$ Tris- $\mathrm{HCl}$, pH 8.0, $5 \mathrm{mM}$ imidazole, $10 \mathrm{mM} \beta$-mercaptoethanol). Lysates in buffer A were mixed with $20 \mu \mathrm{l}$ of Ni-NTA-agarose beads (Qiagen) and incubated at $4{ }^{\circ} \mathrm{C}$ overnight. The beads were then washed sequentially with buffer A, buffer B ( $8 \mathrm{M}$ urea, $0.1 \mathrm{M} \mathrm{Na}_{2} \mathrm{HPO}_{4} / \mathrm{NaH}_{2} \mathrm{PO}_{4}$, pH 8.0, $0.01 \mathrm{M}$ Tris- $\mathrm{HCl}$, pH $8.0,10 \mathrm{mM} \beta$-mercaptoethanol), buffer C (8 M urea, $0.1 \mathrm{M}$ $\mathrm{Na}_{2} \mathrm{HPO}_{4} / \mathrm{NaH}_{2} \mathrm{PO}_{4}, \mathrm{pH} 6.3,0.01 \mathrm{M}$ Tris- $\mathrm{HCl}, \mathrm{pH} 6.3,10 \mathrm{mM}$ $\beta$-mercaptoethanol) $+0.2 \%$ Triton $\mathrm{X}-100$ and buffer $\mathrm{C}$ alone for 5 min each round. Washed beads were then incubated with $40 \mu 1$ elution buffer (200 mM imidazole, $0.15 \mathrm{M}$ Tris-HCl, $\mathrm{pH} 6.7,30 \%$ glycerol, $5 \%$ SDS, $0.72 \mathrm{M} \beta$-mercaptoethanol) at room temperature. The input and the eluates were analyzed by western blotting.

\section{Data collection and statistical analysis}

The intensity of the western blot results was analyzed by densitometry using Bio-Rad Quantity One 4.4.0 software and normal- 
ized to the band with the least intensity, which was arbitrarily set as 1 . The results were expressed as the mean \pm standard deviation (SD). Statistical analysis was performed using the two-tailed, paired Student's $t$ test. A $P$ value of less than 0.05 was considered statistically significant.

\section{Acknowledgments}

This work was supported by the National Institutes of Health (1R01CA131914). This manuscript was also funded in part by the National Natural Science Foundation of China (30811120435, 30870503, 81071657), the Science and Technology Commission of Shanghai Municipality (06DZ22923, 08PJ14047, 10JC1404200, 09ZZ41), and the National Basic Research Program (2009CB918402, 2011CB504200).

\section{References}

1 Chen X, Barton LF, Chi Y, Clurman BE, Roberts JM. Ubiquitin-independent degradation of cell-cycle inhibitors by the REGgamma proteasome. Mol Cell 2007; 26:843-852.

2 Li X, Amazit L, Long W, Lonard DM, Monaco JJ, O’Malley BW. Ubiquitin- and ATP-independent proteolytic turnover of p21 by the REGgamma-proteasome pathway. Mol Cell 2007; 26:831-842.

3 Li X, Lonard D, Jung S, et al. The SRC-3/AIB1 coactivator is degraded in a ubiquitin-and ATP-independent manner by the REG [gamma] proteasome. Cell 2006; 124:381-392.

4 Mao I, Liu J, Li X, Luo H. REGgamma, a proteasome activator and beyond? Cell Mol Life Sci 2008; 65:3971-3980.

5 Roessler M, Rollinger W, Mantovani-Endl L, et al. Identification of PSME3 as a novel serum tumor marker for colorectal cancer by combining two-dimensional polyacrylamide gel electrophoresis with a strictly mass spectrometry-based approach for data analysis. Mol Cell Proteomics 2006; 5:2092.

$6 \mathrm{Yu} \mathrm{G}, \mathrm{Zhao} \mathrm{Y}, \mathrm{He} \mathrm{J}$ et al. Comparative analysis of REG \{gamma expression in mouse and human tissues. $\mathrm{J} \mathrm{Mol}$ Cell Biol 2010; 2:192-198.

7 Yeh ET. SUMOylation and De-SUMOylation: wrestling with life's processes. J Biol Chem 2009; 284:8223-8227.

8 Wang Y, Dasso M. SUMOylation and deSUMOylation at a glance. J Cell Sci 2009; 122:4249.

9 Geiss-Friedlander R, Melchior F. Concepts in sumoylation: a decade on. Nat Rev Mol Cell Biol 2007; 8:947-956.

10 Yurchenko V, Xue Z, Sadofsky M. SUMO modification of human XRCC4 regulates its localization and function in DNA double-strand break repair. Mol Cell Biol 2006; 26:1786.

11 Matunis MJ, Coutavas E, Blobel G. A novel ubiquitin-like modification modulates the partitioning of the Ran-GTPaseactivating protein RanGAP1 between the cytosol and the nuclear pore complex. J Cell Biol 1996; 135:1457-1470.

12 Desterro JM, Rodriguez MS, Hay RT. SUMO-1 modification of IkappaBalpha inhibits NF-kappaB activation. Mol Cell 1998; 2:233-239.

13 Zhang Z, Zhang R. Proteasome activator PA28 gamma regulates $\mathrm{p} 53$ by enhancing its MDM2-mediated degradation. EMBO J 2008; 27:852-864.

14 Rechsteiner M, Hill CP. Mobilizing the proteolytic machine: cell biological roles of proteasome activators and inhibitors. Trends Cell Biol 2005; 15:27-33.

15 Miyauchi Y, Yogosawa S, Honda R, Nishida T, Yasuda H. Sumoylation of Mdm2 by protein inhibitor of activated STAT (PIAS) and RanBP2 enzymes. J Biol Chem 2002; 277:5013150136.

16 Tan JA, Song J, Chen Y, Durrin LK. Phosphorylation-dependent interaction of SATB1 and PIAS1 directs SUMO-regulated caspase cleavage of SATB1. Mol Cell Biol 2010; 30:28232836.

17 Galanty Y, Belotserkovskaya R, Coates J, Polo S, Miller KM, Jackson SP. Mammalian SUMO E3-ligases PIAS1 and PIAS4 promote responses to DNA double-strand breaks. Nature 2009; 462:935-939.

18 Kahyo T, Nishida T, Yasuda H. Involvement of PIAS1 in the sumoylation of tumor suppressor p53. Mol Cell 2001; 8:713718.

19 Lin D, Huang Y, Jeng J, et al. Role of SUMO-interacting motif in Daxx SUMO modification, subnuclear localization, and repression of SUMOylated transcription factors. Mol Cell 2006; 24:341-354.

20 Figueroa-Romero C, Iniguez-Lluhi JA, Stadler J, et al. SUMOylation of the mitochondrial fission protein Drp1 occurs at multiple nonconsensus sites within the B domain and is linked to its activity cycle. FASEB J 2009; 23:3917-3927.

21 Wood LD, Irvin BJ, Nucifora G, Luce KS, Hiebert SW. Small ubiquitin-like modifier conjugation regulates nuclear export of TEL, a putative tumor suppressor. Proc Natl Acad Sci USA 2003; 100:3257-3262.

22 Cheng J, Kang X, Zhang S, Yeh ET. SUMO-specific protease 1 is essential for stabilization of HIF1alpha during hypoxia. Cell 2007; 131:584-595.

23 Liang M, Melchior F, Feng X, Lin X. Regulation of Smad4 sumoylation and transforming growth factor-beta signaling by protein inhibitor of activated STAT1. J Biol Chem 2004; 279:22857-22865.

24 Verger A, Perdomo J, Crossley M. Modification with SUMO. A role in transcriptional regulation. EMBO Rep 2003; 4:137142 .

(Supplementary information is linked to the online version of the paper on the Cell Research website.) 